\title{
Novel Crown Ether and Salen Metal Chelation Driven Molecular Pincers
}

\author{
Aki M. M. Abe, Juho Helaja and Ari M. P. Koskinen* \\ Laboratory of Organic Chemistry, Helsinki University of Technology, P.O.B. 6100, Fi-02015 HUT, \\ Finland \\ ari.koskinen@hut.fi \\ Supporting information
}

General methods. All reactions were carried out under an atmosphere of argon in flame-dried glassware, unless otherwise noted. Non-aqueous reagents were transferred under argon via syringe or cannula and dried prior to use. THF was distilled from $\mathrm{Na}$ /benzophenone ketyl. $\mathrm{CH}_{2} \mathrm{Cl}_{2}$ and $\mathrm{MeOH}$ were distilled from $\mathrm{CaH}_{2}$. Unless otherwise noted, all other solvents and reagents were used as obtained from the supplier. Analytical TLC was performed on Merck silica gel F254 (230-400 mesh) plates and analyzed by UV light or by staining upon heating with DNP solution (4 g 2,4-dinitrophenylhydrazine, $27 \mathrm{~mL} \mathrm{H}_{2} \mathrm{O}, 20 \mathrm{~mL}$ conc. $\mathrm{H}_{2} \mathrm{SO}_{4}, 67 \mathrm{~mL}$ EtOH) or ninhydrin solution ( $1 \mathrm{~g}$ ninhydrin, $0.2 \mathrm{~mL}$ glacial $\mathrm{AcOH}, 100 \mathrm{~mL}$ EtOH). Flash chromatography technique was performed on Merck silica gel 60 (230-400 mesh) and p.a. grade solvents unless otherwise noted. Infrared spectra were measured on a Perkin Elmer Spectrum One FT-IR spectrometer in dichloromethane solution. The ${ }^{1} \mathrm{H}$ and ${ }^{13} \mathrm{C}$ NMR spectra were recorded in a $\mathrm{CDCl}_{3}$ solution on a Bruker Avance $400\left({ }^{1} \mathrm{H} 399.98 \mathrm{MHz} ;{ }^{13} \mathrm{C} 100.59 \mathrm{MHz}\right)$ spectrometer. Chemical shifts are reported in ppm relative to tetramethylsilane $(\delta 0)$ for ${ }^{1} \mathrm{H}$ NMR. For the ${ }^{13} \mathrm{C}$ NMR spectra, the residual $\mathrm{CDCl}_{3}(\delta 77.0)$ was used as the internal standard. High-resolution mass spectrometric data were obtained by the University of Oulu on Micromass LCT spectrometer.

(2,2,6-Trimethyl-4H-benzo[1,3]dioxin-8-yl)-methanol (3). Solid $p$-TsOH (160 mg, $0.85 \mathrm{mmol}, 0.7 \mathrm{~mol} \%)$ was added to a stirred solution of 4-methyl-2,6-bis-(hydroxymethyl)-phenol (20.0 g, $120 \mathrm{mmol}, 100 \mathrm{~mol} \%)$ and 2,2-dimethoxypropane $(23.0 \mathrm{~mL}, 304 \mathrm{mmol}, 253 \mathrm{~mol} \%)$ in acetone $(25.0 \mathrm{~mL})$. The solution was stirred for $3 \mathrm{~h}$ at room temperature, then neutralized by the addition of excess solid sodium bicarbonate. The mixture was concentrated under reduced pressure, diluted with EtOAc, and washed with water (four times). The organic extract was evaporated under reduced pressure, and HOAc $(18.0 \mathrm{~mL}, 50 \mathrm{v} / \mathrm{v}$ aqueous solution) was added to the residue. The mixture was allowed to stand for $30 \mathrm{~min}$ before being diluted with ethyl ether. The organic layer was washed successively with water (2 times), aqueous $\mathrm{NaHCO}_{3}$ (two times), water (two times), and brine and dried. Evaporation under reduced pressure gave the acetonide 3 (23.5 g, 94\%), which was used directly for the next step without further purification. TLC: $R_{f}=0.45$ (50\% EtOAc in cyclohexane, UV). ${ }^{1} \mathrm{H}-\mathrm{NMR}\left(400 \mathrm{MHz}, \mathrm{CDCl}_{3}\right.$ ) $\delta 6.97$ (brs, $\left.1 \mathrm{H}\right), 6.71$ (brs, $\left.1 \mathrm{H}\right), 4.81$ (s, 2H), 4.61 (d, J=4 Hz, 2H), 2.29 (brs, $1 \mathrm{H}, \mathrm{OH}) 2.26(\mathrm{~s}, 3 \mathrm{H}), 1.54(\mathrm{~s}, 6 \mathrm{H}) .{ }^{13} \mathrm{C}-\mathrm{NMR}\left(100 \mathrm{MHz}, \mathrm{CDCl}_{3}\right) \delta 146.8,129.4,128.4,127.8,124.2$, $119.0,99.6,61.3,60.8,24.8,20.5 .^{1}$

2,2,6-Triimethyl-4H-benzo[1,3]dioxine-8-carbaldehyde (4). ${ }^{2}$ (2,2,6-Trimethyl-4H-benzo[1,3]dioxin-8-yl)methanol 3 (1.22 g, $5.86 \mathrm{mmol}, 100 \mathrm{~mol} \%)$ was dissolved in dry $\mathrm{CH}_{2} \mathrm{Cl}_{2}(50 \mathrm{~mL})$ and PCC (1.89 g, 8.79 $\mathrm{mmol}, 150 \mathrm{~mol} \%$ ) was added in one portion. After stirring for $2 \mathrm{~h}$ at $\mathrm{rt}$, the slurry was filtered through Celite and then through a pad of $\mathrm{SiO}_{2}$ to remove the urea byproducts. Removal of the solvent under reduced pressure gave acetonide $4(1.20 \mathrm{~g}, 99 \%)$ as a colorless oil. TLC: $\mathrm{R}_{f}=0.79\left(10 \% \mathrm{MeOH}\right.$ in $\left.\mathrm{CH}_{2} \mathrm{Cl}_{2}, \mathrm{UV} / \mathrm{DNP}\right)$. IR $\left(\mathrm{CH}_{2} \mathrm{Cl}_{2}, \mathrm{~cm}^{-1}\right): 3055,2989,2864,1682,1615,1601 .{ }^{1} \mathrm{H}-\mathrm{NMR}\left(400 \mathrm{MHz}, \mathrm{CDCl}_{3}\right) \delta 10.38(\mathrm{~s}, 1 \mathrm{H}), 7.52$ $(\mathrm{d}, 1 \mathrm{H}, J=1.6 \mathrm{~Hz}), 7.01(\mathrm{~d}, 1 \mathrm{H}, J=1.6 \mathrm{~Hz}), 4.86(\mathrm{~s}, 2 \mathrm{H}), 2.29(\mathrm{~s}, 3 \mathrm{H}), 1.60(\mathrm{~s}, 6 \mathrm{H}) .{ }^{13} \mathrm{C}-\mathrm{NMR}(100 \mathrm{MHz}$, $\left.\mathrm{CDCl}_{3}\right) \delta 189.2,152.1,131.5,129.4,126.8,123.9,120.2,100.4,60.6,24.8,20.4$. HRMS (ESI) calcd for $\mathrm{C}_{12} \mathrm{H}_{14} \mathrm{NaO}_{3} 229.0841[\mathrm{M}+\mathrm{Na}]^{+}$, found 229.0867 . 
3-Bromomethyl-5-methyl-2-hydroxy-benzaldehyde (5). In a two-neck round bottom flask fitted with a gas inlet and outlet, acetonide $4(1.1 \mathrm{~g}, 5.33 \mathrm{mmol}, 100 \mathrm{~mol} \%)$ was dissolved in dry hexanes $(40 \mathrm{~mL}) . \operatorname{HBr}(\mathrm{g})$ (generated by dropping $\mathrm{H}_{2} \mathrm{SO}_{4}(13.7 \mathrm{~mL}, 256 \mathrm{mmol}, 5000 \mathrm{~mol} \%$ ) into $\mathrm{NaBr}(27 \mathrm{~g}, 267 \mathrm{mmol}, 5000 \mathrm{~mol} \%$ ) in a second two-neck round bottom flask equipped with an addition funnel and a gas outlet to the reaction flask) was bubbled into the reaction solution for approximately $15 \mathrm{~min}$, after which the pink solution was stirred at $\mathrm{rt}$ for $30 \mathrm{~min}$. The solution was then passed through a plug of $\mathrm{Na}_{2} \mathrm{SO}_{4}$ and concentrated. The crude product was dissolved in $\mathrm{CH}_{2} \mathrm{Cl}_{2}$ and filtered through a pad of $\mathrm{SiO}_{2}$. Removing the solvent under reduced pressure gave benzyl bromide $\mathbf{5}(1.21 \mathrm{~g}, 98 \%)$ as a white cotton wool like solid. TLC: $R_{f}=0.52(10 \% \mathrm{MeOH}$ in $\mathrm{CH}_{2} \mathrm{Cl}_{2}$, UV/DNP). mp 111-112 ${ }^{\circ} \mathrm{C}$. IR $\left(\mathrm{CH}_{2} \mathrm{Cl}_{2}, \mathrm{~cm}^{-1}\right): 3059,2926,2854,2746,1658,1619,1608,1467$ $\mathrm{cm}^{-1} .{ }^{1} \mathrm{H}-\mathrm{NMR}\left(400 \mathrm{MHz}, \mathrm{CDCl}_{3}\right) \delta 11.30(\mathrm{~s}, 1 \mathrm{H}, \mathrm{OH}), 9.86(\mathrm{~s}, 1 \mathrm{H}), 7.43(\mathrm{~d}, 1 \mathrm{H}, J=1.6 \mathrm{~Hz}), 7.33(\mathrm{~d}, 1 \mathrm{H}, J$ $=1.6 \mathrm{~Hz}), 4.56(\mathrm{~s}, 2 \mathrm{H}), 2.34(\mathrm{~s}, 3 \mathrm{H}) .{ }^{13} \mathrm{C}-\mathrm{NMR}\left(100 \mathrm{MHz}, \mathrm{CDCl}_{3}\right) \delta 196.4,157.4,138.8,134.2,129.3,126.0$, 120.5, 26.7, 20.2. HRMS (ESI) calcd for $\mathrm{C}_{9} \mathrm{H}_{10} \mathrm{O}_{2} \mathrm{Br}[\mathrm{M}+\mathrm{H}]^{+} 228.9864$, found 228.9881 .

3,3'-(2,2'-(1,2-phenylenebis(oxy))bis(ethane-2,1-diyl))bis(oxy)bis(methylene)bis(2-hydroxy-5-

methylbenzaldehyde) (7). 1,2-bis(2-hydroxyethoxy)benzene 6 (98 mg, $0.5 \mathrm{mmol}, 100 \mathrm{~mol} \%$ ) was dissolved in THF (5 mL) and NaH (88 mg, $2.2 \mathrm{mmol}, 440 \mathrm{~mol} \%, 60 \mathrm{wt}-\%$ dispersion in mineral oil) was added. The solution was stirred for $1 \mathrm{~h}$ at $\mathrm{rt}$. Benzyl bromide 5 (280 mg, $1.2 \mathrm{mmol}, 240 \mathrm{~mol} \%$ ) in THF (5 mL) was cannulated slowly into the reaction mixture. The mixture was stirred at $60{ }^{\circ} \mathrm{C}$ for $2 \mathrm{~h}$. After cooling, the solution was diluted with $\mathrm{CH}_{2} \mathrm{Cl}_{2}$, washed with water and brine. Drying, filtration and evaporation under reduced pressure gave the crude product, which was purified by silica gel column chromatography to yield 7 (52 mg, 21\%) as a yellowish amorphous solid. TLC: $R_{f}=0.85\left(5 \% \mathrm{MeOH}\right.$ in $\left.\mathrm{CH}_{2} \mathrm{Cl}_{2}, \mathrm{UV} / \mathrm{DNP}\right)$. IR $\left(\mathrm{CH}_{2} \mathrm{Cl}_{2}\right.$, $\mathrm{cm}^{-1}$ ): 3062, 2930, 2869, 1655, 1623, 1607, 1505, 1459. ${ }^{1} \mathrm{H}-\mathrm{NMR}\left(400 \mathrm{MHz}, \mathrm{CDCl}_{3}\right) \delta 11.03$ (brs, 2H, OH), $9.81(\mathrm{~s}, 2 \mathrm{H}), 7.45(\mathrm{~d}, 2 \mathrm{H}, J=1.7 \mathrm{~Hz}), 7.22(\mathrm{~d}, 2 \mathrm{H}, J=1.7 \mathrm{~Hz}), 6.97-6.90(\mathrm{~m}, 4 \mathrm{H}), 4.66(\mathrm{~s}, 4 \mathrm{H}), 4.23(\mathrm{dd}, 4 \mathrm{H}$, $\left.J_{1}=4.3 \mathrm{~Hz}, J_{2}=5.6 \mathrm{~Hz}\right), 3.91\left(\mathrm{dd}, 4 \mathrm{H}, J_{1}=4.3 \mathrm{~Hz}, J_{2}=5.6 \mathrm{~Hz}\right), 2.29(\mathrm{~s}, 6 \mathrm{H}) .{ }^{13} \mathrm{C}-\mathrm{NMR}\left(100 \mathrm{MHz}, \mathrm{CDCl}_{3}\right) \delta$ 196.4, 156.9, 149.0, 137.2, 132.5, 128.8, 126.6, 121.6, 119.9, 115.0, 69.3, 68.9, 66.9, 20.2. HRMS (ESI) calcd for $\mathrm{C}_{28} \mathrm{H}_{30} \mathrm{O}_{8} \mathrm{Na}[\mathrm{M}+\mathrm{Na}]^{+}$517.1838, found 517.1825.

(E)-6,6'-(2,2'-(1,2-phenylenebis(oxy))bis(ethane-2,1-diyl))bis(oxy)bis(methylene)bis(4-methyl-2-((E)(pyren-1-ylmethylimino)methyl)phenol) (8). Aldehyde 7 (110 mg, $0.22 \mathrm{mmol}$, a00 mol \%) and 1pyrenemethylamine $(103 \mathrm{mg}, 0.44 \mathrm{mmol}, 200 \mathrm{~mol} \%)$ were dissolved in a mixture of $\mathrm{MeOH}(10 \mathrm{~mL})$ and toluene $(6.5 \mathrm{~mL})$. The resulting solution was heated at $65{ }^{\circ} \mathrm{C}$ overnight. The solvent was evaporated under reduced pressure. Silica gel column chromatography yielded the crown-salen-pyrene $\mathbf{8}(160 \mathrm{mg}, 78 \%)$ as a yellow foam. TLC: $R_{f}=0.95$ (5\% MeOH in $\mathrm{CH}_{2} \mathrm{Cl}_{2}$, UV/ninhydrin). IR $\left(\mathrm{CH}_{2} \mathrm{Cl}_{2}, \mathrm{~cm}^{-1}\right): 3064,3042,2929$, 2868, 1634, 1606, 1505, 1464. ${ }^{1} \mathrm{H}-\mathrm{NMR}\left(400 \mathrm{MHz}, \mathrm{CDCl}_{3}\right) \delta 13.49$ (brs, 2H, OH), 8.3-7.9 (m, 18H), 7.18 (d, $2 \mathrm{H}, J=1.6 \mathrm{~Hz}), 6.92-6.85(\mathrm{~m}, 4 \mathrm{H}), 6.83(\mathrm{~d}, 2 \mathrm{H}, J=1.6 \mathrm{~Hz}), 5.43(\mathrm{~s}, 4 \mathrm{H}), 4.63(\mathrm{~s}, 4 \mathrm{H}), 4.18\left(\mathrm{dd}, 4 \mathrm{H}, J_{1}=4.4\right.$ $\left.\mathrm{Hz}, J_{2}=5.6 \mathrm{~Hz}\right), 3.85\left(\mathrm{dd}, 4 \mathrm{H}, J_{1}=4.4 \mathrm{~Hz}, J_{2}=5.6 \mathrm{~Hz}\right), 2.30(\mathrm{~s}, 6 \mathrm{H}) .{ }^{13} \mathrm{C}-\mathrm{NMR}\left(100 \mathrm{MHz}, \mathrm{CDCl}_{3}\right) \delta 165.56$, $156.57,149.13,132.86,131.24,131.21,131.00,130.82,130.74,128.82$, 128.09, 127.36, 127.34, 127.24, 126.79, 125.98, 125.70, 125.30, 125.24, 124.92, 124.86, 124.72, 122.87, 121.54, 118.07, 115.14, 68.99, 67.59, 60.63, 29.70, 20.30. HRMS (ESI) calcd for $\mathrm{C}_{62} \mathrm{H}_{52} \mathrm{~N}_{2} \mathrm{O}_{6} \mathrm{Na}[\mathrm{M}+\mathrm{Na}]^{+} 943.3723$, found 943.3751 .

\section{References}

1. Tsubaki, K.; Otsubo, T.; Tanaka, K.; Fuji, K. J. Org. Chem. 1998, 63, 3260-3256.

2. DiMauro, E. F.; Kozlowski, M. C. Org. Lett. 2001, 3, 3053. 


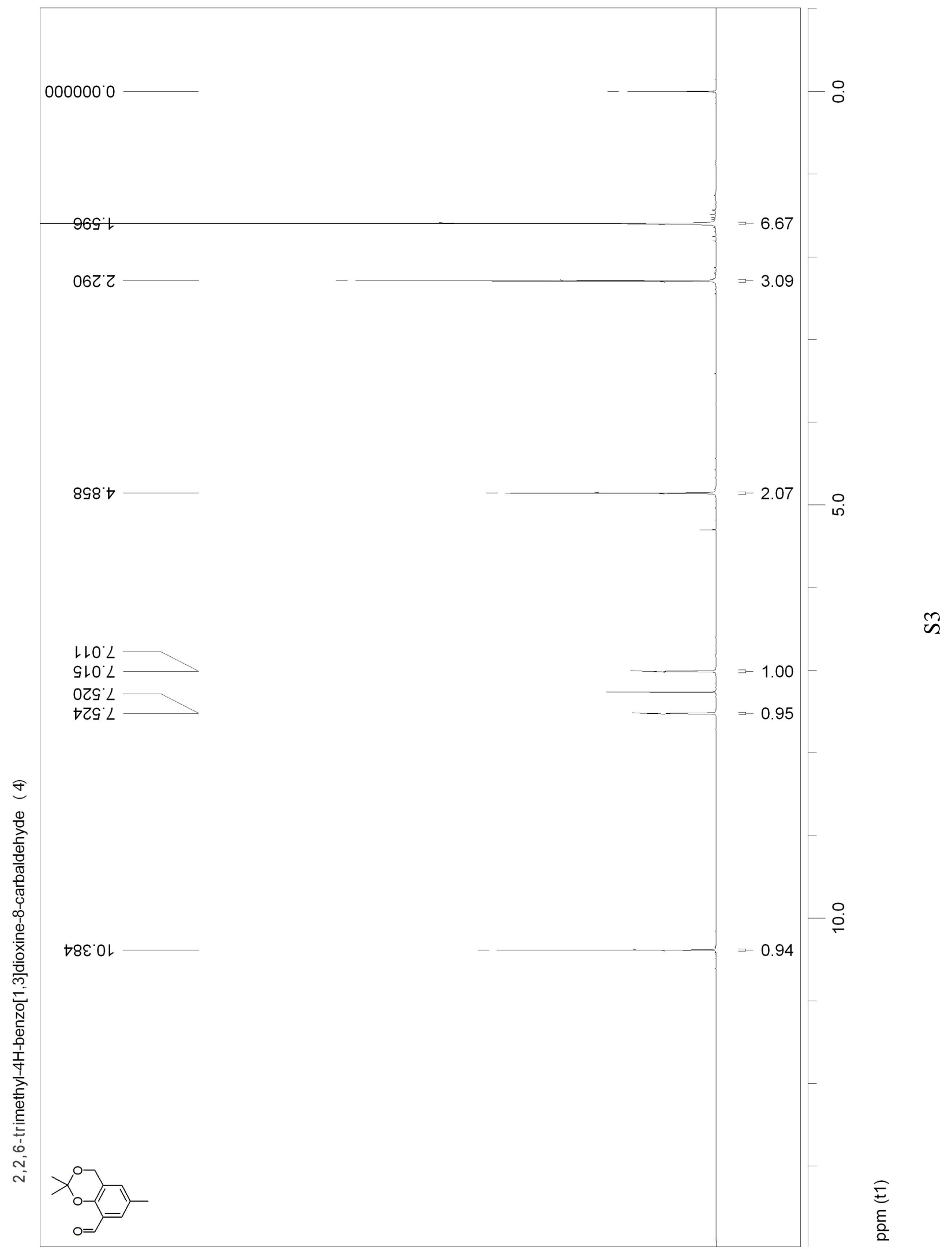




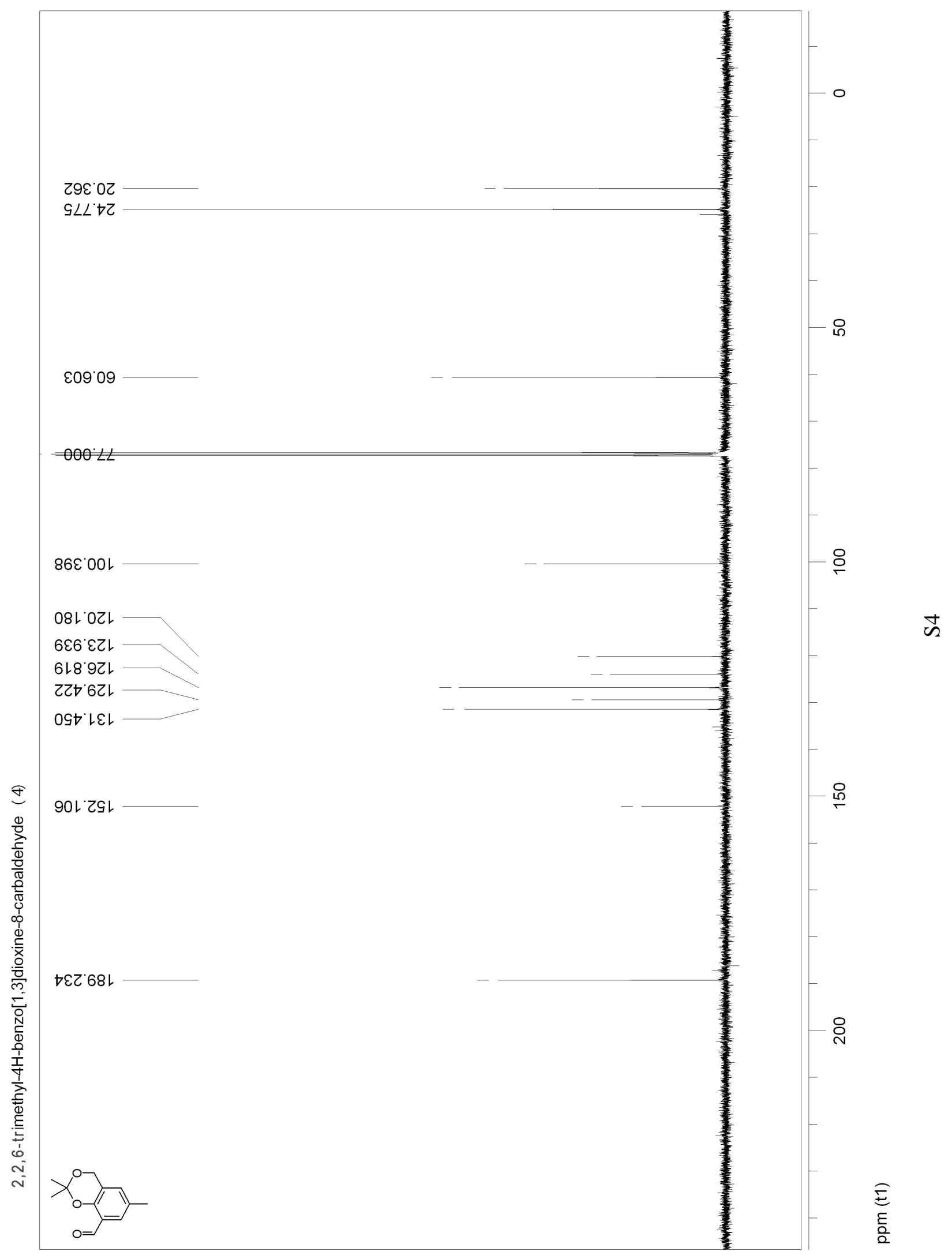




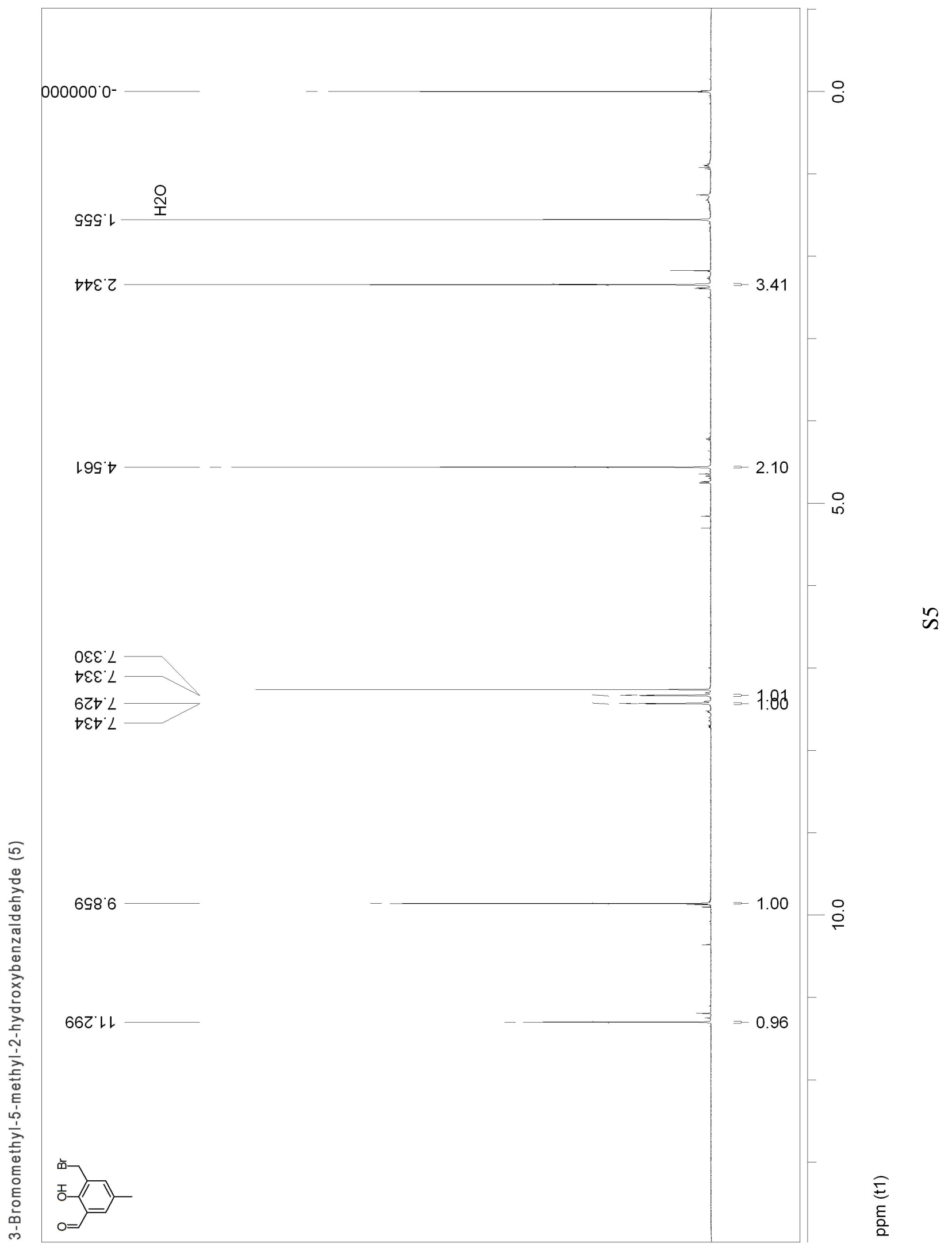




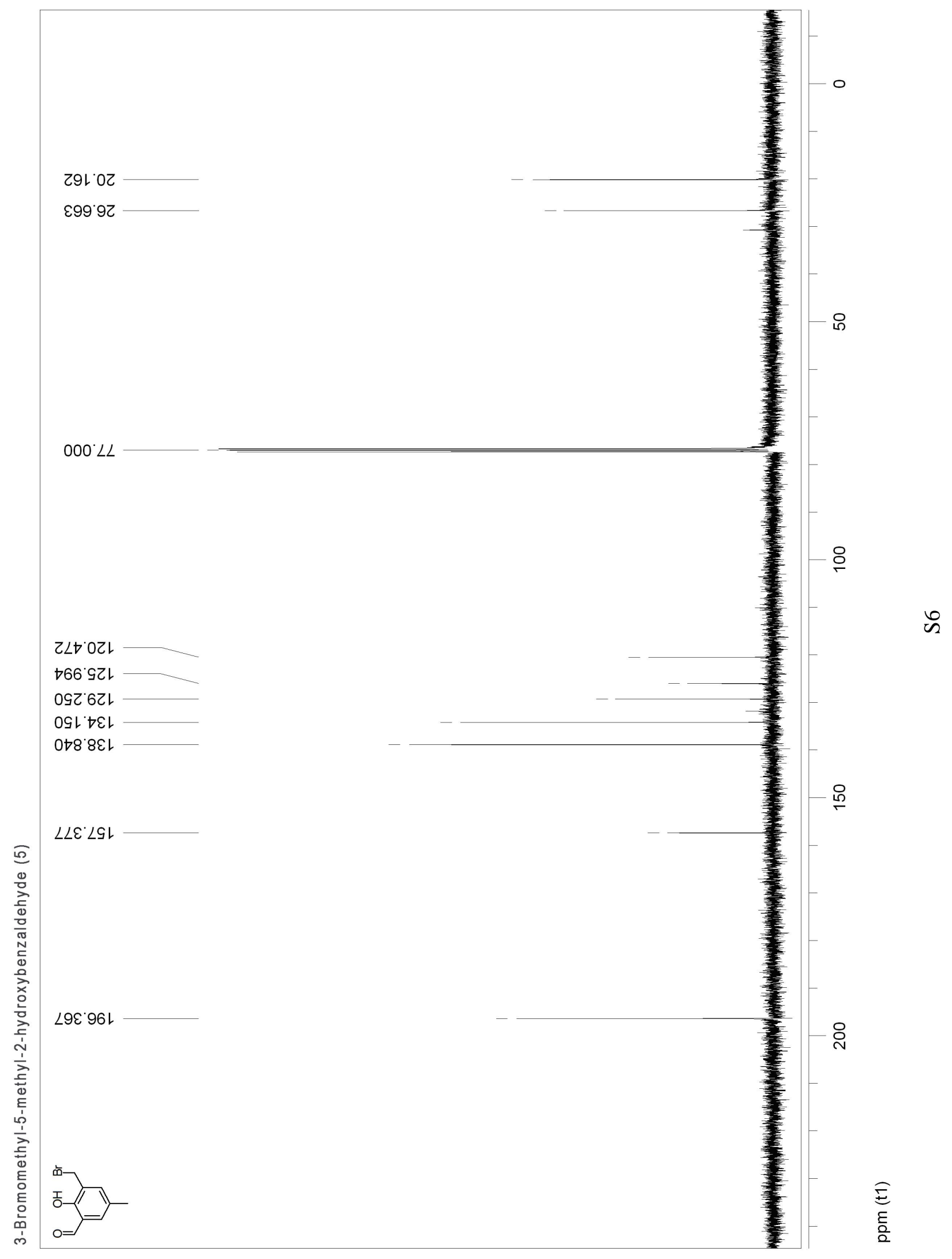




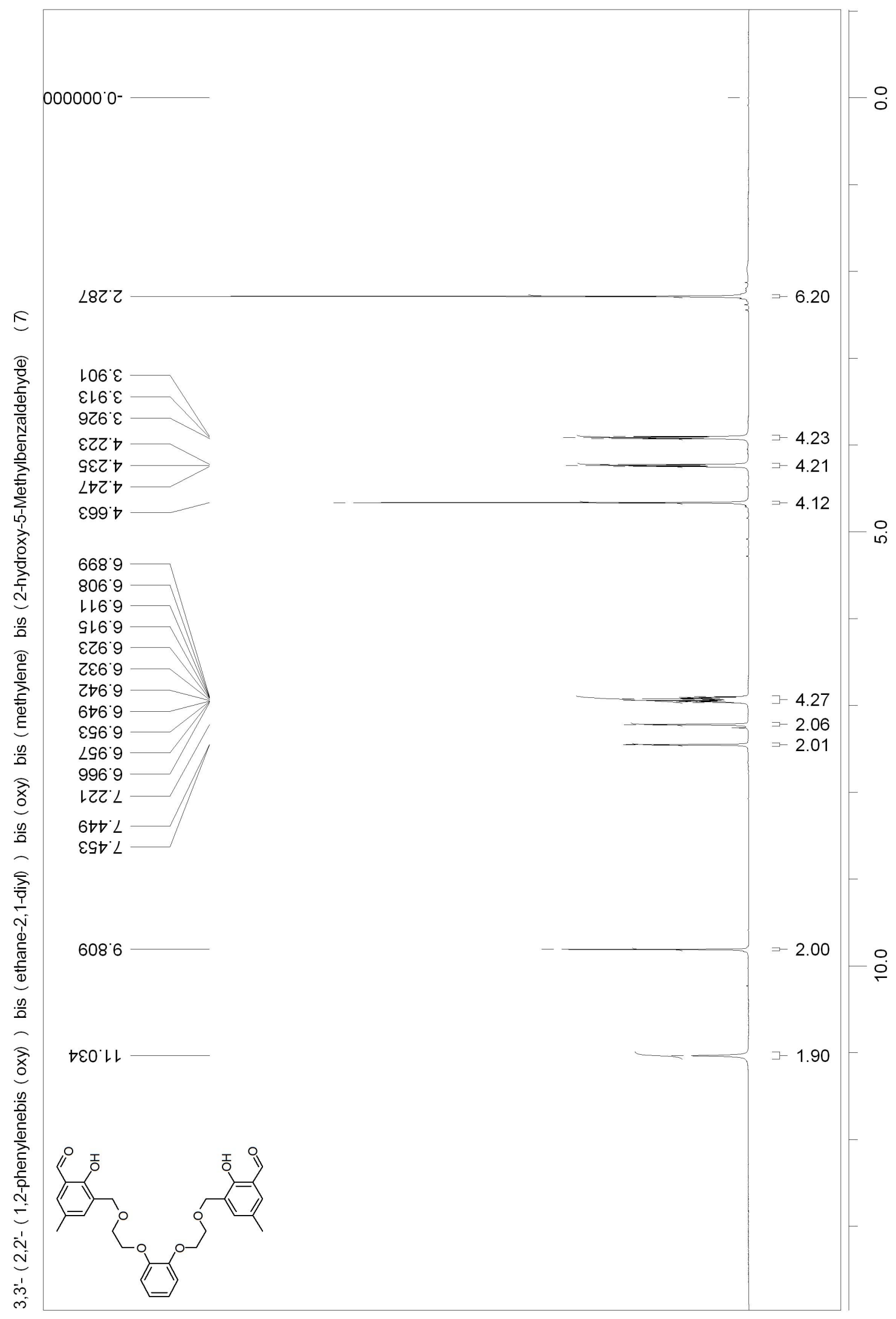

is 


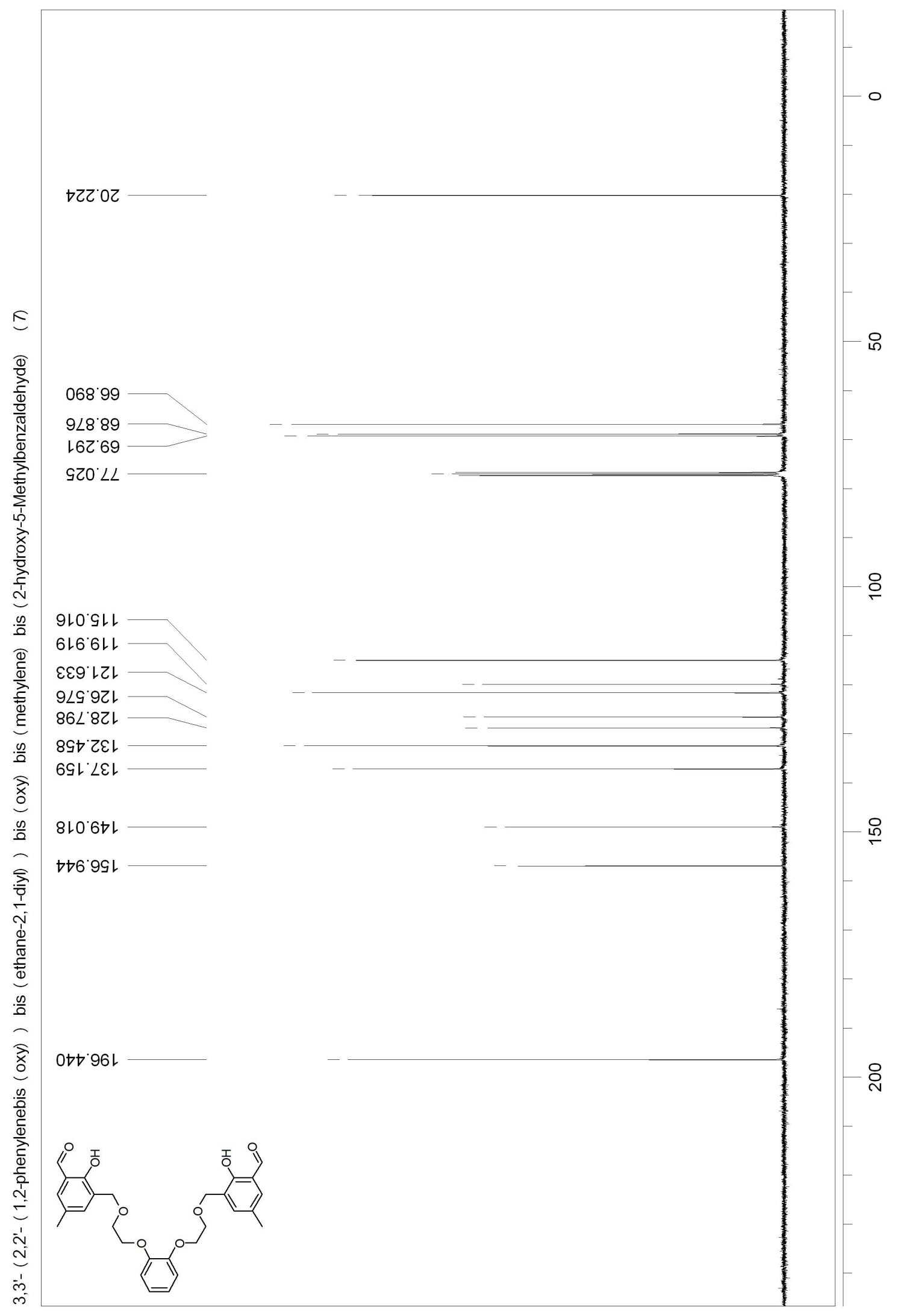

$\infty$

$\underset{\text { 흥 }}{E}$ 


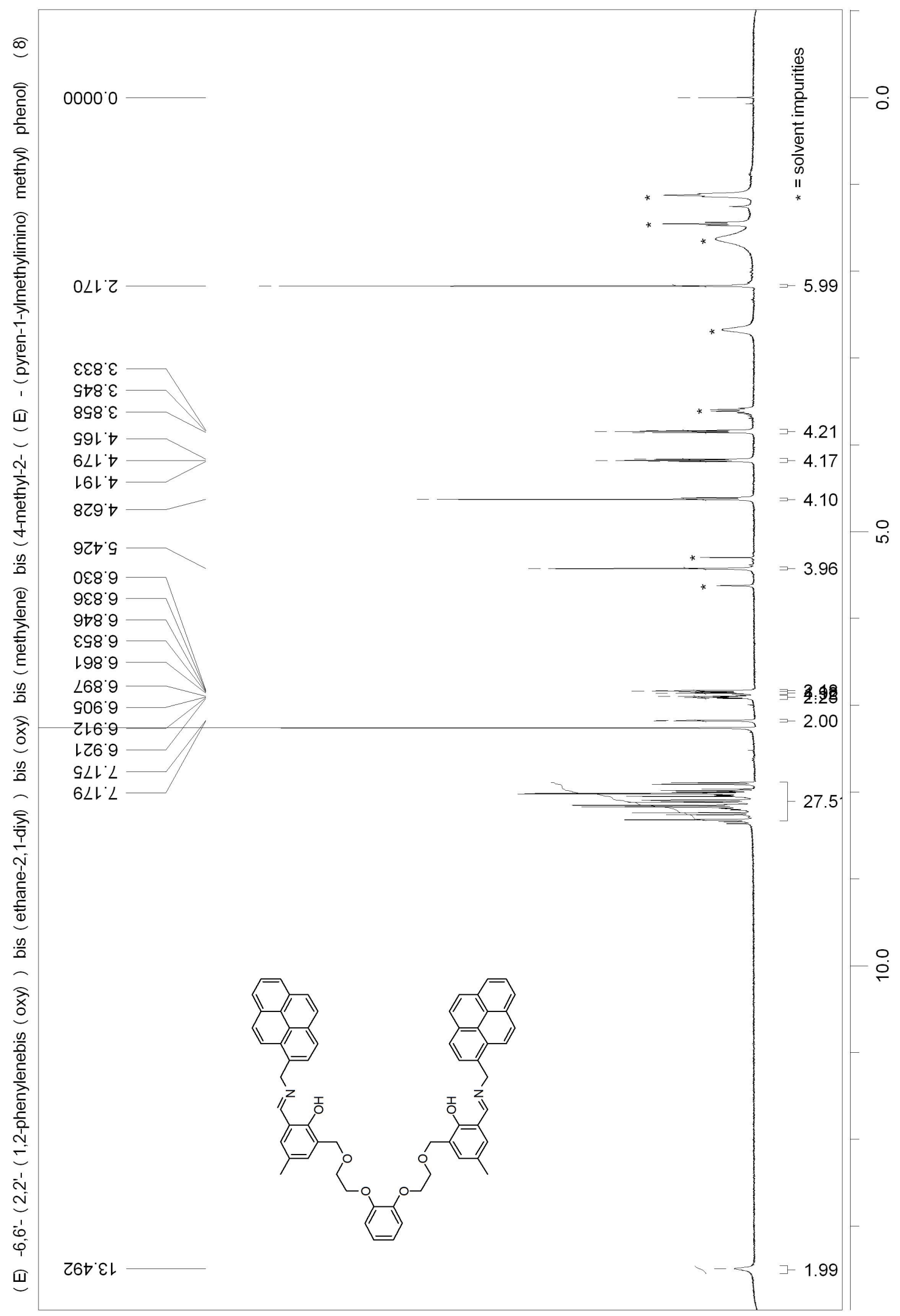

के

E
흘 


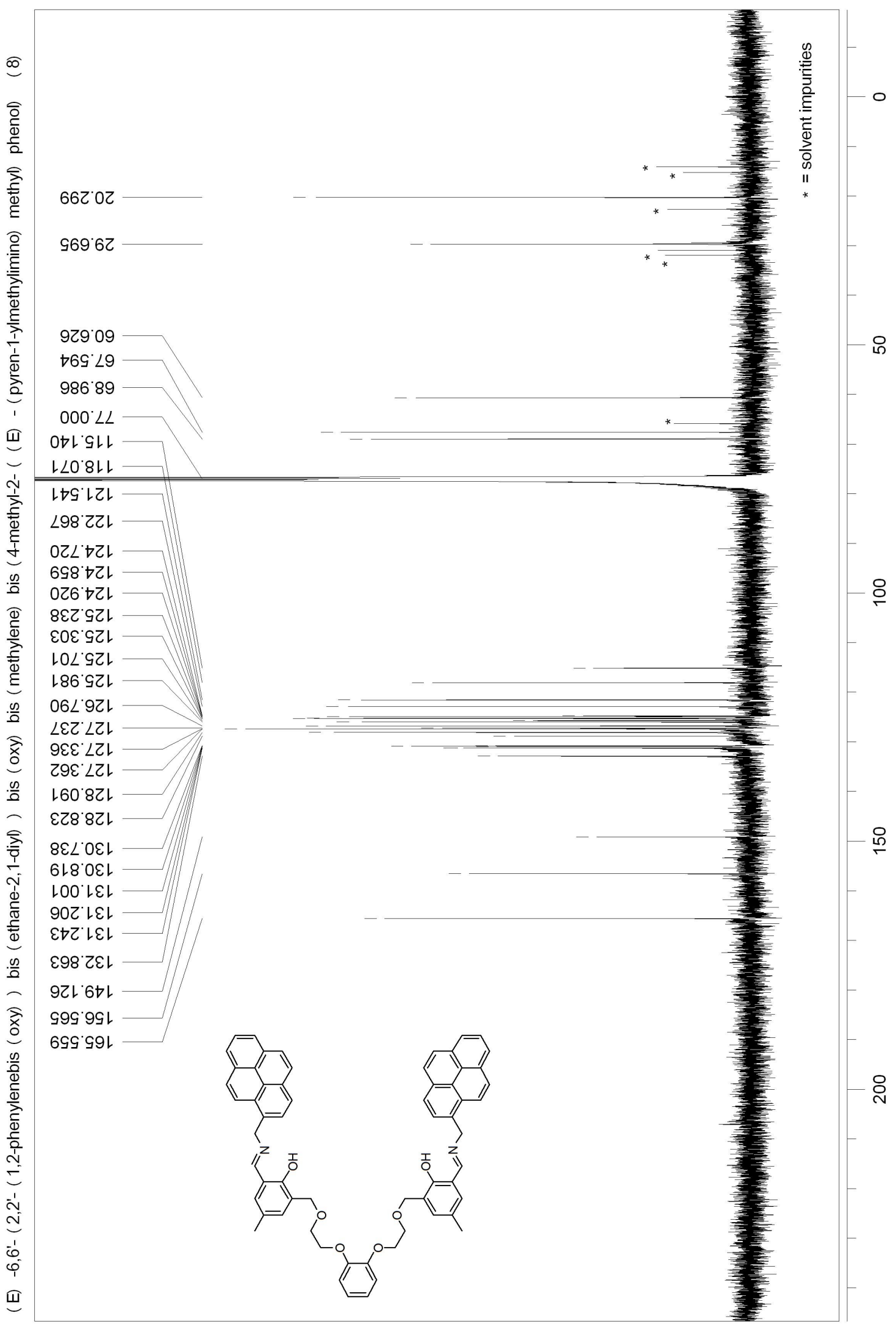

$\frac{0}{n}$ 